\title{
A Comparative Analysis of Forgery Detection Algorithms
}

\author{
Davide Cozzolino, Giovanni Poggi, Carlo Sansone, and Luisa Verdoliva \\ Department of Electrical Engineering and Information Technologies \\ University Federico II of Naples \\ Via Claudio, 21, 80125 Naples, Italy \\ \{davide.cozzolino, poggi, carlosan, verdoliv\}@unina.it
}

\begin{abstract}
The aim of this work is to make an objective comparison between different forgery techniques and present a tool that helps taking a more reliable decision about the integrity of a given image or part of it. The considered techniques, all recently proposed in the scientific community, follow different and complementary approaches so as to guarantee robustness with respect to tampering of different types and characteristics. Experiments have been conducted on a large set of images using an automatic copy-paste tampering generator. Early results point out significant differences about competing techniques, depending also on complexity and side information.
\end{abstract}

Keywords: forgery detection, digital forensics, image tampering.

\section{Introduction}

With the ever increasing diffusion of simple and powerful software tools for digital source editing, image tampering is becoming more and more common, stimulating an intense quest for algorithms, to be used in the forensics field, which help deciding about the integrity of digital images. In particular, passive techniques draw the highest attention as they require no collaboration on the part of the user through some types of watermarks or signatures.

A large variety of approaches have been proposed in the literature, which take advantage of the inconsistencies (e.g., in the histogram or in the sampling grid) arising from the various tampering types. Following [1, they can be classified as pixel-based, format-based, camera-based, and phisics/geometric-based.

In extreme synthesis, pixel-based techniques analyze the correlation between pixels either directly in the spatial domain or in some transformed domain. Format-based methods, instead, exploit the usual adoption of some lossy compression scheme, like JPEG, which introduces some recognizable marks in the presence of manipulations. Camera-based techniques take advantage of features specific of any different camera models, and even of individual cameras, to use them as image signatures to exploit in forgery detection. Finally, phisics/ geometric-based methods study higher-level inconsistencies between the imaged scene/object and the forgery source, such as illumination, object size, etc. 
The many existing approaches, which comprise in turn a very large and ever growing number of individual detection techniques, testify both the interest towards this problem and its complexity. In fact, no ultimate solution exists to the image forgery detection problem. Each technique is based on some important hypotheses which limit its applicability, and therefore it is always possible to find cases where it fails. On the other hand, one should take for granted that a malicious tamperer, aware of the principles on which each technique works, will be able to trick it, given enough time and resources. Having a multiplicity of different tools is therefore essential to guarantee a high probability of detecting forgeries.

Given the great variety of approaches, comparing forgery detection algorithms turns out to be a very challenging task, although a much needed one. To increase confusion, many Authors, to support the claim of good performance of their proposed algorithm, provide results on a restricted set of images, without facing the problem of making an extensive comparison with other approaches in terms of key performance indices. So, the habit of proposing more and more new algorithms seems to prevail against the need of assessing the performance of the existing ones in an objective way.

In this work we perform an experimental performance comparison of a limited but significant set of forgery detection techniques. A similar work was presented in [2] where several techniques were considered, all belonging to the format-based category. Here, we consider four techniques chosen among the most popular and promising in the recent literature, belonging to different categories: pixel-based, format-based, and camera-based. As dataset, we used a set of images taken from a standard database [3] while forgeries have been obtained by using the copypaste approach proposed in [4]. Tests have been carried out by varying the size and statistics of the tampered area, and results are reported in terms of the usual sensitivity and specificity parameters.

All algorithms are implemented in Matlab and integrated into an easy-to-use software tool, written in JAVA, easily extensible to include new techniques and functionalities. The tool allows one to process one or more images at once, select one or more detection algorithms, so as to allow comparison of results, and look for a forgery on the whole image or else only in a part of it.

In the following section we describe briefly the forgery detection algorithms under comparison, while in section three the experimental setting is presented and results are commented. Finally section 4 draws conclusions and outlines future work.

\section{Forgery Techniques under Analysis}

For this comparative study, in order to sample solutions as different as possible, we have chosen techniques that use various approaches: format based [5], pixelbased [6] and camera-based [7] [8]. In the following we will briefly describe the implemented algorithms.

Format-based methods take advantage of the specific format of images. Since most images are JPEG compressed, to detect a tampering it is possible to exploit 
the blocking effect introduced by JPEG, which gives rise to the so-called Block Artifact Grid (BAG).

In fact, manipulating images in this format causes an alteration of these artifacts, mainly in the case of copy and paste processing, since the BAG of the original image and that of the copied region very likely mismatch. In [5] a simple method is proposed to identify this type of forgery, named here Li-2009 after the name of the first Author and the year of publication, a convention followed for the other techniques as well. The basic idea is to extract the horizontal and vertical edges due to JPEG artifacts by means of a second order derivative followed by a thresholding operation in order to eliminate edges relative to signal discontinuities. A further enhancement is then realized to obtain the block artifact grid. If the image has been subject to a copy-paste processing a BAG mismatching can be detected when lines are present within a $8 \times 8$ block. The procedure delineated in [5] tries to determine this presence through summations along rows and columns both inside and at the boundaries of the block.

In 6] a pixel-based technique is proposed, Popescu-2005, relying on finding traces of resampling in the image. The idea is based on the observation that tampering may alter the underlying statistics. In fact, when an image is modified, operations like resizing, rotating and stretching must be typically performed, which require to resample the original image. This process introduces correlations that, once detected, can be considered as evidence of a digital tampering. The detection process is based on estimating, through the expectation/maximization algorithm, a set of periodic samples that are correlated to their neighbors.

A very powerful approach in detecting forgeries relies on artifacts introduced by the digital camera itself, and in particular the photo-response non uniformity (PRNU) which can be considered as a sort of intrinsic fingerprint of a specific digital camera. The PRNU arises from differences and imperfections in the silicon wafer used to manufacture the imaging sensor: these physical differences provide a unique sensor fingerprint which can be used for forgery detection. The Chen-2008 algorithm, proposed in [7, requires the preliminary estimation of the camera PRNU from a large number of images taken by the camera itself. Then, the PRNU of the image under investigation is estimated and compared with the reference. This step is quite challenging, since this fingerprint is much weaker than the image, therefore a denoising step is used, which removes much of the image content increasing the signal-to-noise ratio. In [9] we replaced the original denoising algorithm with state-of-the-art nonlocal filtering, obtaining a significant performance improvement. The PRNU comparison is carried out by sliding an analysis window of dimension $128 \times 128$ over the image: if the camera PRNU is present, the block (or more correctly its central pixel) is labeled as genuine, otherwise it is considered tampered. The test statistic used for detection is the normalized correlation value with a decision threshold selected so as to obtain the required false acceptance rate. A similar algorithm, Zhang-2010, has been recently proposed in [8]. It makes use of canonical correlation analysis (CCA) to measure the linear correlation between the two PRNU estimates. Only for heavily textured areas, identified in advance in the image, a Neyman-Pearson decision is used like in [7. 


\section{Experimental Results}

We now study the performance of the selected forgery detection techniques through simulation experiments on a large number of tampered images.

Our test set is formed starting from 72 high-resolution photos of the Dresden Image Database [3. Each image is then subject to copy-paste forgery by means of the Photoshop scripts of the University of Catania 4, which allows to superpose objects taken from a small library, scaled and translated at will, over the target image. Some photos have been used several times with different forgeries, reaching a total of 108 images, presenting one or more forgeries. Relatively large forgeries are considered, cover about $20 \%$ of the image area, because most techniques become too unreliable in the presence of small tampered regions. PRNU-based techniques, for example, discard altogether dubious objects smaller than $64 \times 64$ pixels, although a recent segmentation-based version [10] allows one to deal also with somewhat smaller objects. Fig.1 shows one of the original images together with its tampered version.
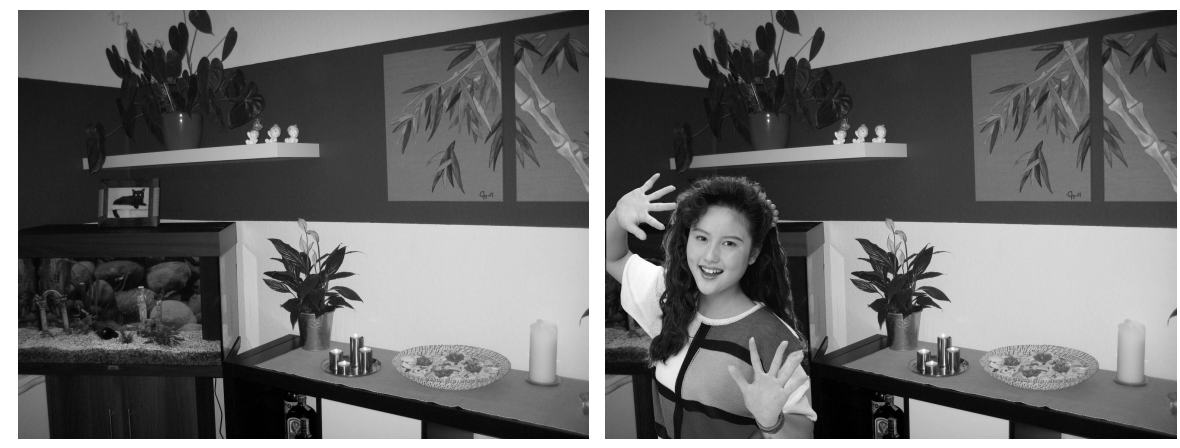

Fig. 1. An original test image (left) and its tampered version (right)

The selected techniques, as described in the literature, are not immediately comparable since they provide different types of results: some are pixel-based, some block-based, some require visual inspection. In order to provide homogeneous results, the image is divided in non-overlapping square blocks, of size $128 \times 128$ through $1024 \times 1024$ pixels, with decisions taken independently for each of them considered as a whole.

For each technique we compute on the entire database the quantities

TP (true positive): \# forged blocks declared forged

FP (false positive): \# genuine blocks declared forged

$T N$ (true negative): \# genuine blocks declared genuine

$F N$ (false positive): \# forged blocks declared genuine 
Results are then given in terms of sensitivity, specificity and accuracy, computed as

$$
\begin{gathered}
\text { sensitivity }=\frac{T P}{T P+F N} \\
\text { specificity }=\frac{T N}{T N+F P} \\
\text { accuracy }=\frac{T N+T P}{T N+F P+T P+F N}
\end{gathered}
$$

which measure, respectively, the ability to detect the presence of forgery, the ability to confirm the absence of forgery, and the overall classification accuracy, independent of the nature of the blocks.

Figures 2 through 5 report results for block-sizes $128 \times 128,256 \times 256,512 \times 512$, and $1024 \times 1024$.

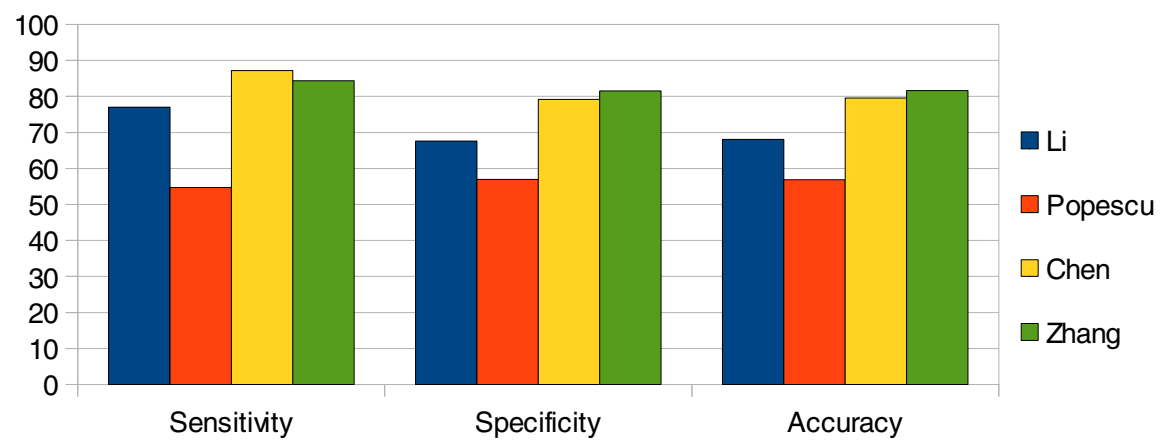

Fig. 2. Results for blocks of size $128 \times 128$

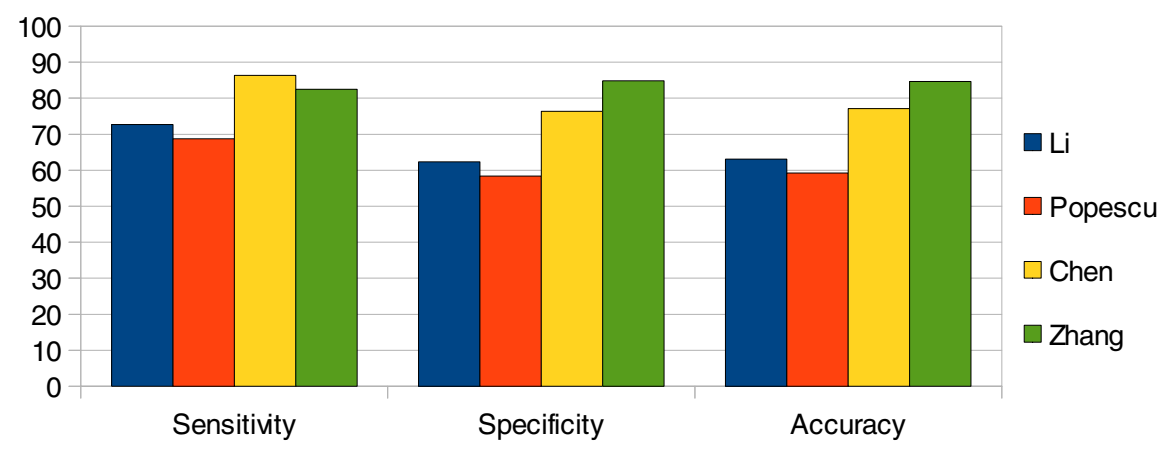

Fig. 3. Results for blocks of size $256 \times 256$ 


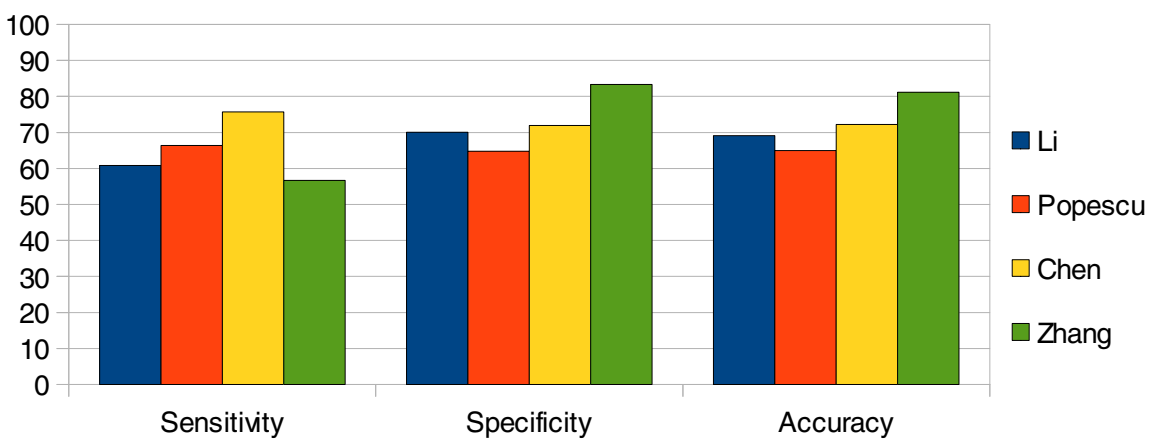

Fig. 4. Results for blocks of size $512 \times 512$

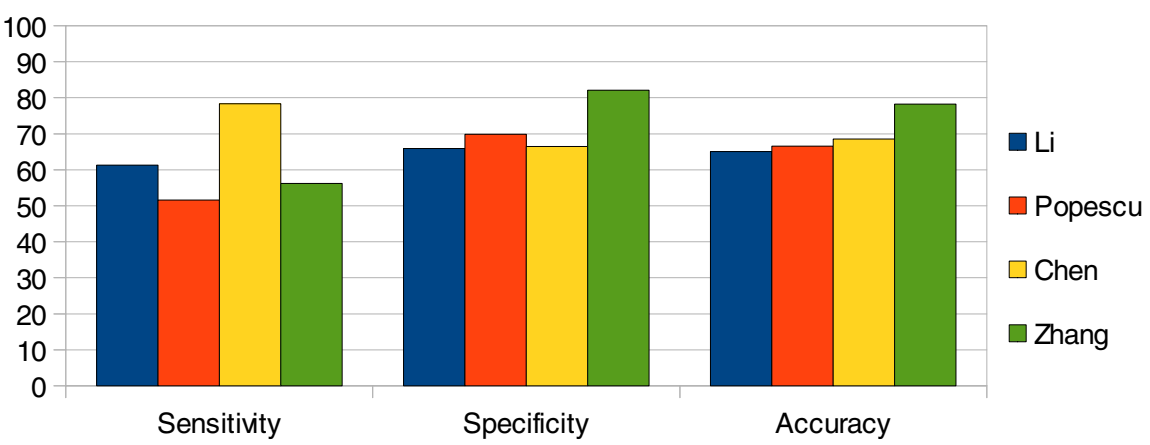

Fig. 5. Results for blocks of size $1024 \times 1024$

The first obvious consideration is that camera-based techniques, Chen-2008 and Zhang-2010, perform generally much better than the others, under all points of view. On the other hand, these techniques rely on an important piece of information, the PRNU, obtained by means of significant pre-processing. In addition, they are applicable only if the camera or a collection of photos taken by it are available, a requirement not always met in applications. The gap between camera-based and other techniques reduces when larger blocks are considered, probably for the increased number of blocks that are only partially forged, for which all decisions become quite arbitrary. This is obviously a limit of the experimental protocol, but also of the block-based nature of most algorithms.

Turning to the non-camera-based algorithms, Li-2009 seems to perform slightly better than Popescu-2005, in general, with a more significant gain when small blocks are considered. This latter quality can be important in the presence of small-size forgeries which are intrinsically more difficult to detect and therefore more challenging for a forgery detection algorithm.

As a visual example of results, Fig. 6 shows the output provided for the image of Fig. 1 by the Chen-2008 and Li-2009 methods, where only the $128 \times 128$ blocks 
considered tampered are shown. As expected, the camera-based Chen-2008 is clearly more accurate. In this case, however, the bad performance of Li-2009 is also a consequence of the low compression ratio of the original JPEG image, which therefore shows little or no trace of the block artifact grid needed to detect forgeries. This gives us the opportunity to underline once again that no single technique works in all cases and a judicious use of several complementary techniques is always recommended.
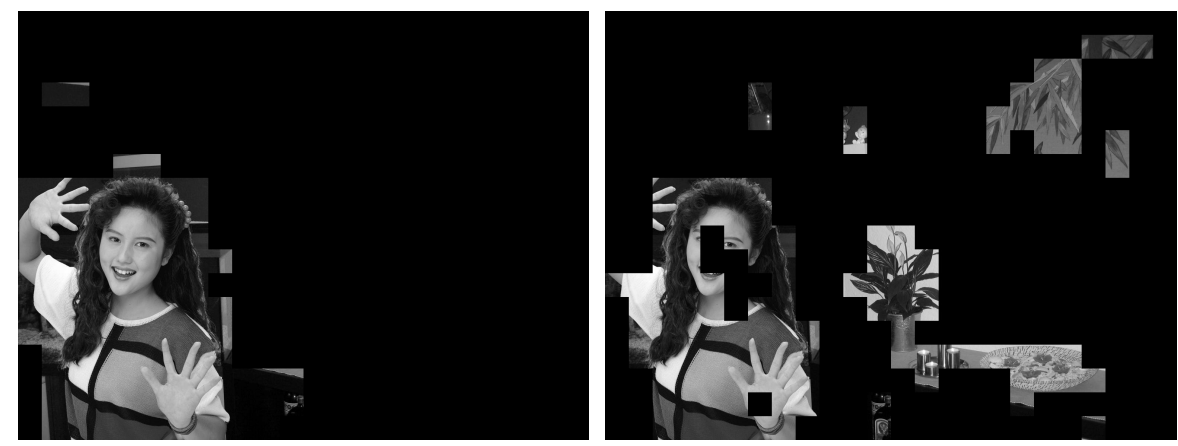

Fig. 6. Results of Chen-2008 (left) and Li-2009 algorithms for the image of Fig.1

\section{Conclusions and Future Work}

In this work we dealt with the challenging task of comparing several forgery detection techniques. Experimental results are quite reasonable, as performance seems to depend strongly on complexity and side information available. Obviously, we want is to extend this type of comparison including many more techniques, a work which is currently under way.

The major problems encountered, besides the implementation of the individual techniques, concern the interpretation of their results in terms of homogeneous and meaningful performance measures. Under this point of view, the block-based approach represents just a reasonable compromise, upon which we are already trying to improve.

Another important goal is to improve the overall detection reliability by implementing an information fusion level in which the output of many different detection algorithm is taken into account and properly combined. Some preliminary experiments have been conducted by using a simple weighted sum of the individual decisions [11. Although only a very small set of detectors is available, results are already encouraging.

\section{References}

1. Farid, H.: Image forgery detection. IEEE Signal Processing Magazine 26, 16-25 (2009) 
2. Battiato, S., Messina, G.: Digital Forgery Estimation into DCT Domain - A Critical Analysis. In: ACM Multimedia Workshop on Multimedia in Forensics, pp. 37-42 (2009)

3. Gloe, T., Böhme, R.: The Dresden Image Database for Benchmarking Digital Image Forensics Categories and Subject Descriptors. In: ACM Symposium on Applied Computing, pp. 1584-1590 (2010)

4. IPLab. Image Processing Laboratory - Forensic Database, http://iplab.dmi.unict.it/index .php?option=comdocman\&Itemid=111

5. Li, W., Yuan, Y., Yu, N.: Passive detection of doctored JPEG image via block artifact grid extraction. Signal Processing 89(9), 1821-1829 (2009)

6. Popescu, A.C., Farid, H.: Exposing digital forgeries by detecting traces of resampling. IEEE Transactions on Signal Processing 53(2), 758-767 (2005)

7. Chen, M., Fridrich, J., Goljan, M., Lukas, J.: Determining Image Origin and Integrity Using Sensor Noise. IEEE Transactions on Information Forensics and Security 3, 74-90 (2008)

8. Zhang, C., Zhang, H.: Exposing Digital Image Forgeries by Using Canonical Correlation Analysis. In: International Conference on Pattern Recognition (ICPR), pp. 838-841 (2010)

9. Chierchia, G., Parrilli, S., Poggi, G., Sansone, C., Verdoliva, L.: On the influence of denoising in PRNU based forgery detection. In: 2nd ACM Workshop on Multimedia in Forensics, Security and Intelligence (MiFOR), pp. 117-122 (2010)

10. Chierchia, G., Parrilli, S., Poggi, G., Sansone, C., Verdoliva, L.: PRNU-based detection of small size image forgeries. In: 17th International Conference on Digital Signal Processing (DSP), pp. 1-6 (2011)

11. Kuncheva, L.I.: Combining Pattern Classifiers: Methods and Algorithms. Wiley Interscience (2004) 